\title{
Surgical Approaches for Tibial Plateau Fractures
}

\author{
Utku Kandemir, MD ${ }^{1}$ Jeffrey Maclean, MS, MD ${ }^{1}$ \\ ${ }^{1}$ Department of Orthopaedic Surgery, University of California San \\ Francisco, San Francisco, California \\ J Knee Surg 2014;27:21-30.
}

\begin{abstract}
Address for correspondence Utku Kandemir, MD, Department of Orthopaedic Surgery, University of California San Francisco, Orthopaedic Trauma Institute, 2550 23rd Street, Building 9, 2nd Floor, San Francisco, CA 94110 (e-mail: kandemiru@orthosurg.ucsf.edu).
\end{abstract}

\begin{abstract}
Keywords

- tibial plateau fracture

- surgical approach

- fibular osteotomy

Tibial plateau fractures are common yet complex injuries, and include a wide variety of fracture patterns. They are also potentially associated with significant soft tissue injury. Thus, the surgeon preparing to operate on one of these fractures must choose from a variety of surgical approaches that have been described. The choice of approach is dictated principally by the fracture pattern, with consideration of the soft tissue envelope, patient factors, and associated injuries. Occasionally, associated intra-articular injuries may also need to be addressed. Commonly used approaches to the proximal tibia, which together allow for the treatment of any proximal tibia fracture, will be described in this article.
\end{abstract}

Tibial plateau fractures are common injuries. They can occur in any age group but have a bimodal distribution with highenergy injuries usually occurring in young adults with good bone quality and low-energy fractures that typically occur in elderly patients with poor bone quality. They are mainly associated with axial, bending, and rotational forces or combination of these.

Understanding the degree of soft tissue injury around the knee is of utmost importance regarding the timing of surgical intervention unless a purely percutaneous approach will be utilized. Although the reduction is technically easier when performed earlier, surgical delay may be necessary to decrease the risk of wound complications.

The ideal surgical approach should provide exposure of the fracture site that is sufficient to facilitate both reduction of the fracture and application of the fixation implants, with minimal risk of complications such as wound healing or infection. Careful dissection and atraumatic soft tissue handling is necessary to avoid neurovascular injury or further iatrogenic insult to soft tissues. The choice of approach is dictated principally by the fracture pattern, with consideration of the soft tissue envelope, patient factors, and associated injuries (e.g., need for vascular repair or fasciotomies) occasionally altering the surgical plan. The use of computed tomography (CT) has greatly improved our ability to understand the pattern of injury. ${ }^{1}$

received

November 3, 2013

accepted

November 4, 2013

published online

December 19, 2013
CT scan with sagittal and coronal reconstructions is obtained in all proximal tibia fractures. If temporizing external fixation is necessary, CT scan is deferred until after closed reduction and external fixation has been performed. A detailed analysis of the fracture pattern, especially with respect to fracture plane(s), displacement of the joint surface, and comminution is necessary to develop a surgical tactic for fracture reduction and application of fixation implants, which in turn is critical in deciding the surgical approach(es) to be used.

Early techniques for the treatment of proximal tibial fractures relied on a direct anterior midline approach, which required large soft tissue flaps to access the corresponding fracture. ${ }^{2,3}$ To address associated collateral ligament and meniscal injuries, a modification using an inverted "L"-type incision over the joint line was described. ${ }^{4}$ Over time, the importance of soft tissue management and the correlation of outcomes with anatomic reduction have led to fracturespecific approaches. The diminishing use of peripatellar incisions, descriptions of the posteromedial and posterolateral approaches, and common use of a dual-approach technique are the principle evolutions since Tscherne and Lobenhoffer published their overview 20 years ago. ${ }^{5}$ Commonly used approaches to the proximal tibia, which together allow for the treatment of any proximal tibia fracture, will be described in this article.
Copyright ( $\odot 2014$ by Thieme Medical Publishers, Inc., 333 Seventh Avenue, New York, NY 10001, USA. Tel: +1(212) 584-4662.
DOI http://dx.doi.org/ 10.1055/s-0033-1363519. ISSN $1538-8506$. 


\section{General Considerations}

A fully radiolucent table (Mizuho OSI, Union City, CA) is used in all cases. This allows radiographic evaluation in multiple planes including a joint line view (anteroposterior [AP] view tilted 10 degrees caudally to be parallel to the posterior slope of the tibial plateau), oblique views, a true lateral view, and views in line with the fracture lines that are taken to assess fracture reduction and fixation. A nonsterile tourniquet is placed as high on the thigh as possible. For a posterior approach, a sterile tourniquet is applied to ensure that the proximal aspect of the skin incision is not draped out of the surgical field. In unicondylar fractures, a bump under the hip and torso is utilized tilting the patient approximately 30 degrees to have access for the exposure in the resting position. The bump is applied on the ipsilateral side in lateral plateau fractures and on the contralateral side in medial plateau fractures. In bicondylar fractures either a small bump or no bump is used because access to both medial and lateral sides of the leg is necessary. A triangle or bump placed under the leg helps to avoid the contralateral leg while obtaining the lateral view with the C-arm. The universal distractor is an extremely useful tool and it is used in the majority of cases to improve the visualization and the quality of reduction. Reduction clamps, bone tamps, a lamina spreader, minifragment set, Kirschner wires, and bone void filler (cancellous allograft or other type of fillers) are planned in addition to the definitive fixation implant, most commonly a precontoured periarticular plate.

\section{Percutaneous Approaches}

Pure split (Schatzker type I) or pure depression (Schatzker type III) fracture patterns may be amenable to percutaneous approaches. Timing of surgical intervention may be as early as the day of injury as incisions are limited to 1 to $2 \mathrm{~cm}$. Appropriate fluoroscopic imaging is of paramount importance in percutaneous surgery as both the adequacy of reduction and positioning of fixation implants relies on fluoroscopy.

For pure split fractures the reduction is achieved using periarticular clamps with ball-spike tips. The prongs of the clamps are placed at the medial and lateral side 1 to $2 \mathrm{~cm}$ below the joint line through small incisions. Disc attachments may be used to increase the surface area of the tips of the clamps while applying compression, especially in osteoporotic bones. Then, the reduction is confirmed using fluoroscopic imaging in both AP and lateral joint line views. Multiple guide wires for appropriate cannulated screws are placed adjacent to the subchondral bone and parallel to the joint line. Fixation is usually accomplished with multiple (at least three) 6.5/7.0-mm lag screws. Washers may be used to avoid penetration of the screw head into the bone thus increasing surface area of compression of screws.

For pure depression type of fractures, the goal is to elevate the depressed portion of the joint surface to the anatomic level. A 2- to $3-\mathrm{cm}$ incision is created at the anterolateral proximal tibia usually 4 to $5 \mathrm{~cm}$ below the joint line. A bone window of $1-\mathrm{cm}$ diameter is created using a $2.0-$ or $2.5-\mathrm{mm}$ drill bit and a chisel to connect the drill holes. Alternatively, a bone tamp can be used to create a window on the cortex by pushing the cortex into the metaphyseal area with a mallet. Bone tamps are then used to elevate the depressed joint surface. It is critical to check the location of the fracture in both AP and lateral fluoroscopic views to correctly place the bone tamp under the depressed joint surface as relying on one view can easily be misleading. Undue force should be avoided while using bone tamps, as inadvertent entry into the joint is a pitfall of this technique. The void created by the reduction of the depressed joint surface and the tract used by the bone tamp is filled with cancellous allograft or a bioabsorbable cement to support the elevated joint surface. Then, multiple 3.5-mm cortical screws are placed percutaneously using a "rafter" technique to further support the reduction.

In addition to fluoroscopic imaging, arthroscopy is a tool that can be useful for direct visualization of the joint surface and assessment of the accuracy of reduction. One should be aware of extravasating fluid used during arthroscopy through the fracture site into the leg compartments, potentially resulting in compartment syndrome. Therefore, repeated examination of the leg throughout the surgical intervention and postoperatively is warranted, as well as limiting the amount of time that arthroscopy done, and using gravity for fluid inflow instead of an irrigation pump.

On the basis of the surgeon's preference, pure split (Schatzker type I) and pure depression (Schatzker type III) fractures can also be treated with open anterolateral approach. In our experience, open approaches provide more consistent reduction and fixation in the split-depressed facture pattern (Schatzker type II) and bicondylar fracture variants (Schatzker type V and type VI).

\section{Anterolateral Approach}

Because most tibial plateau fractures involve the lateral tibial plateau, an anterolateral approach is the most frequently used approach for the treatment of plateau fractures (-Figs. 1-4). This approach is typically utilized for splitdepression lateral plateau (Schatzker type II) and bicondylar (Schatzker type VI, type V) fracture patterns. When an unstable tubercle fragment is encountered, a separate small anterior incision can be added for direct reduction and fixation of the tubercle.

\section{Superficial Dissection}

Two slight variations in the skin incision can be used. The incision starts from 2 to $3 \mathrm{~cm}$ proximal to the joint line and extends $3 \mathrm{~cm}$ below the inferior margin of the tibial tubercle, and can be extended as far distally as needed. The lazy "S"shaped incision starts direct lateral over the iliotibial (IT) band, curves over Gerdy tubercle (GT), and continues distally 1-cm lateral to the tibial crest. Alternatively, a gentle curvilinear incision centered over GT can be created. Starting at the center of GT, the IT band is cut in line with the fibers proximally. Distal to the GT, the fascia of the anterior compartment of the leg is incised in continuity with the IT band 


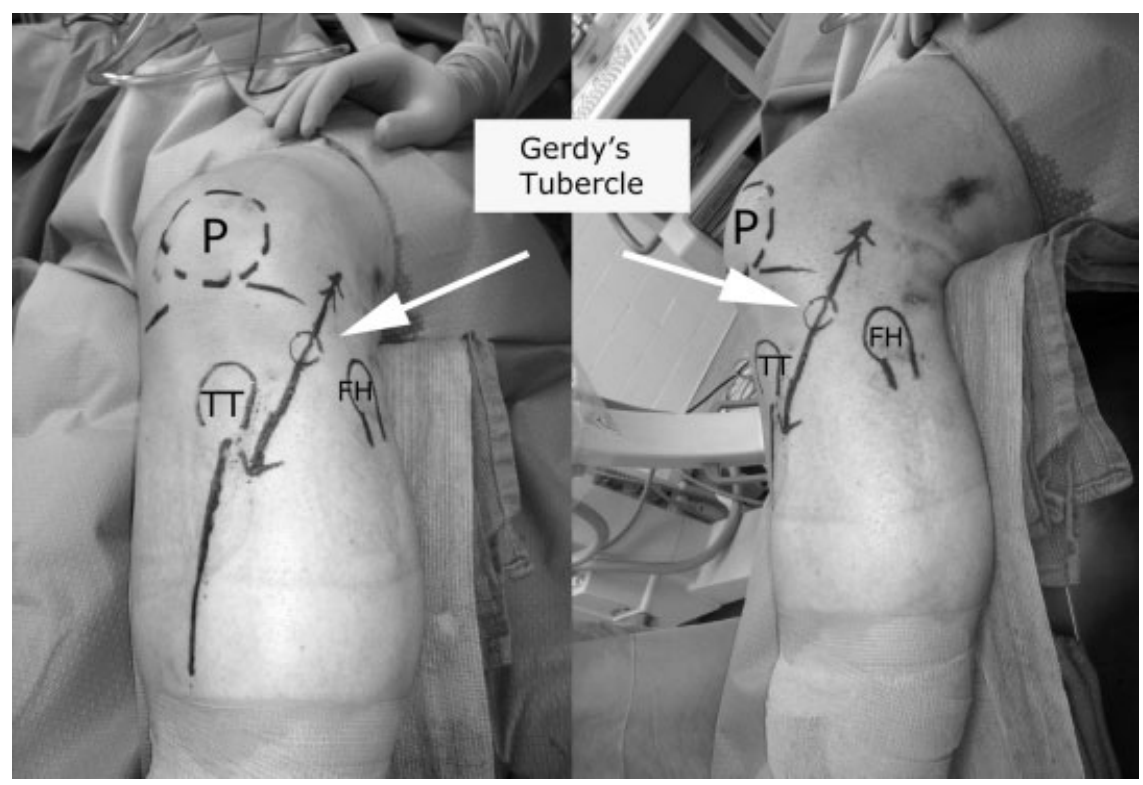

Fig. 1 Anterolateral approach: The incision starts 2 to $3 \mathrm{~cm}$ proximal to the joint line crossing the Gerdy tubercle and aiming $1 \mathrm{~cm}$ off the lateral border of the tibial crest extends $3 \mathrm{~cm}$ below the inferior margin of the tibial tubercle, and can be extended as far distally as needed. P, patella; TT, tibial tuberosity; $\mathrm{FH}$, fibular head.

incision, aiming toward the lateral border of the tibial tubercle and tibial crest. The IT band is detached from its insertion using sharp dissection with a knife and reflected anteriorly and posteriorly. The interval between the IT band and the joint capsule is developed with blunt dissection and care is taken to keep the capsule intact. Distally, the anterior compartment fascia is incised.

If visualization of the joint (i.e., a submeniscal arthrotomy) is not planned, a hockey-stick skin incision can be utilized for minimally invasive application of plate fixation. The proximal limb of the incision is parallel to the joint line, curving over the GT and aiming distally $1 \mathrm{~cm}$ off the lateral border of the tibial crest.

\section{Deep Dissection}

The origin of the tibialis anterior muscle is reflected from the proximal lateral tibia and retracted posteriorly exposing the anterolateral surface of the proximal tibia. The joint line is identified by direct palpation. A submeniscal arthrotomy is created to directly visualize the articular cartilage. Leaving a cuff of tissue on the tibial side for repair, the meniscotibial ligaments are incised along the tibial border similar to that described by Padanilam et $\mathrm{al}^{6}$ but without dividing the anterior horn of the meniscus. Three or four sutures are placed through the peripheral meniscus in a vertical fashion and used to apply retraction to improve the visualization of the articular surface. These sutures are then used for direct repair to the aforementioned cuff of tissue on the tibial side. Alternatively, they are passed through the small K-wire holes in the periarticular plate before securing the plate to bone and the sutures are tied over the plate. If a peripheral vertical lateral meniscal tear exists, which are commonly associated with joint depression fracture patterns, multiple sutures are passed in vertical fashion through the inner part of the meniscus and through the capsule, thus incorporating the meniscal repair into the repair of the submeniscal arthrotomy.

To aid in direct visualization of the articular surface, a universal distractor is applied with either $5.0-$ or $6.0-\mathrm{mm}$ Schanz pins depending on the size of the bone. The femoral pin is placed 1 to $2 \mathrm{~cm}$ proximal to the lateral epicondyle at the level of metaphyseal flare. The tibial pin is placed 1 to $2 \mathrm{~cm}$ beyond the distal extent of plate that will be applied for fixation. Planning of the length of the plate to be used before the placement of the tibial Schanz pin is important so that the distractor does not interfere with application of definitive fixation. This is determined by selecting the appropriate plate and obtaining a fluoroscopy image with the plate overlying the leg. The bar of the distractor is usually placed posteriorly. When an external fixator has already been placed as part of a staged-treatment protocol, the Schanz pins of the external fixator can be used for application of the universal distractor.

\section{Additional Exposure}

The incision can be extended distally and used to decompress the anterior and lateral compartments of the leg, or for fixation of fracture extension into the tibial shaft if needed.

\section{Medial Approach}

Here, we differentiate medial and posteromedial approaches. The medial approach is used for isolated fractures of the medial plateau (Schatzker type IV) and as part of the "dual incision approach" for bicondylar fractures (- Fig. 5) ${ }^{7-9}$ This approach is appropriate when the unstable fragment is the anterior part of the joint and the fracture line is parallel to the anteromedial surface of the tibia or approximately in the coronal plane. The fixation is placed on the anteromedial surface of the tibia therefore having the screws crossing the fracture line perpendicularly. 


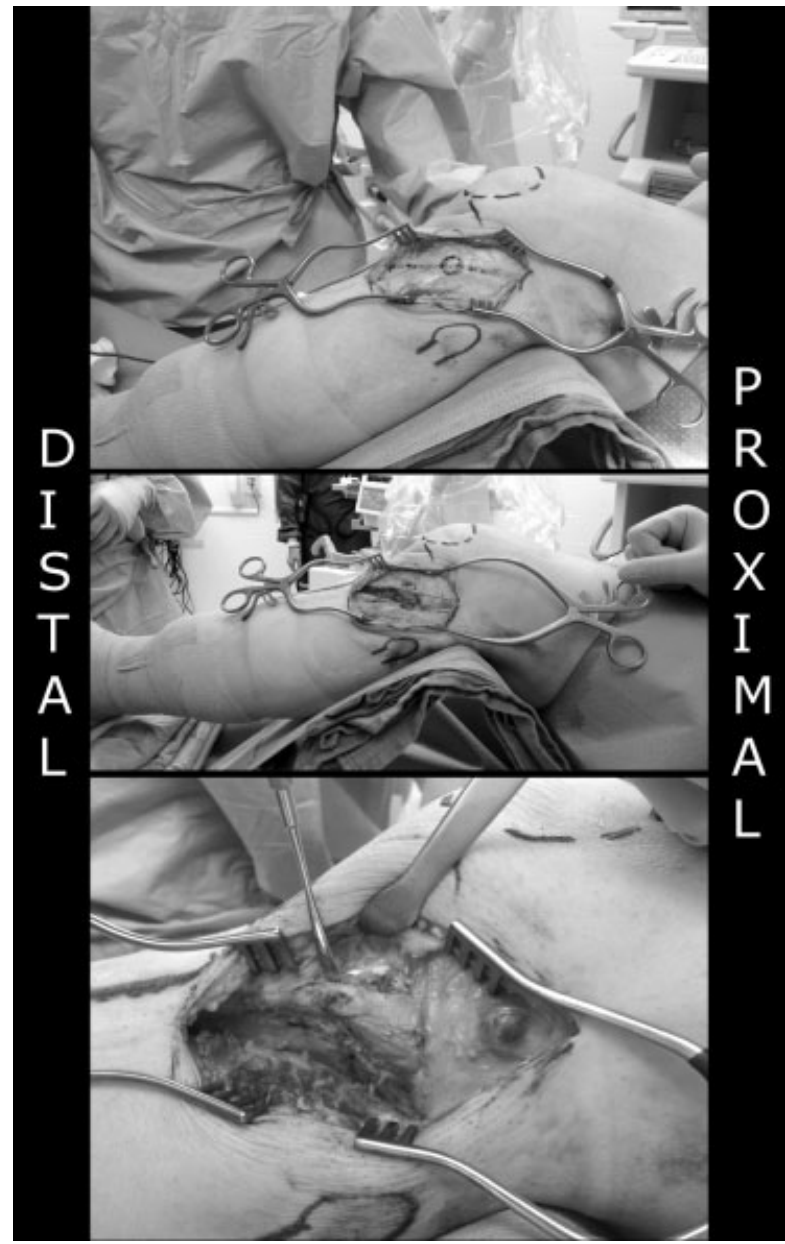

Fig. 2 Anterolateral approach: (Top) Retraction of the skin and subcutaneous tissue exposing the Gerdy tubercle (GT), iliotibial (IT) band, and fascia of the anterolateral leg. (Middle) The IT band and leg fascia are incised in line with skin incision and kept in continuity, detaching the IT band off GT sharply. (Bottom) With anterior and posterior retraction of IT band leg fascia and reflection of the origin of tibialis anterior from the proximal tibia, the split fracture and capsule is exposed.

\section{Superficial Dissection}

The skin incision starts 1 to $2 \mathrm{~cm}$ proximal to the joint line in line with the medial femoral epicondyle and extends over the pes anserinus insertion, bisecting the tibial crest and the posteromedial border of the proximal tibia. The length of the incision is based on the metaphyseal extent of the fracture. The saphenous nerve and vein are generally posterior to this incision but caution should be used, as anatomic variations exist. With the knee bent 15 degrees, a straight incision is made 10 to $15 \mathrm{~cm}$ in length. Warren et al described the medial knee structures to be in three layers. ${ }^{10}$ Just deep to the subcutaneous tissues, the sartorius fascia is encountered and incised in line with the skin incision. Distally, the gracilis and semitendinosus tendons are identified coming from posterior to their insertion on the anteromedial tibia.

\section{Deep Dissection}

The pes anserinus tendons are skeletonized proximally and distally. This exposes the second layer, which contains the superficial medial collateral ligament (MCL). If necessary, the

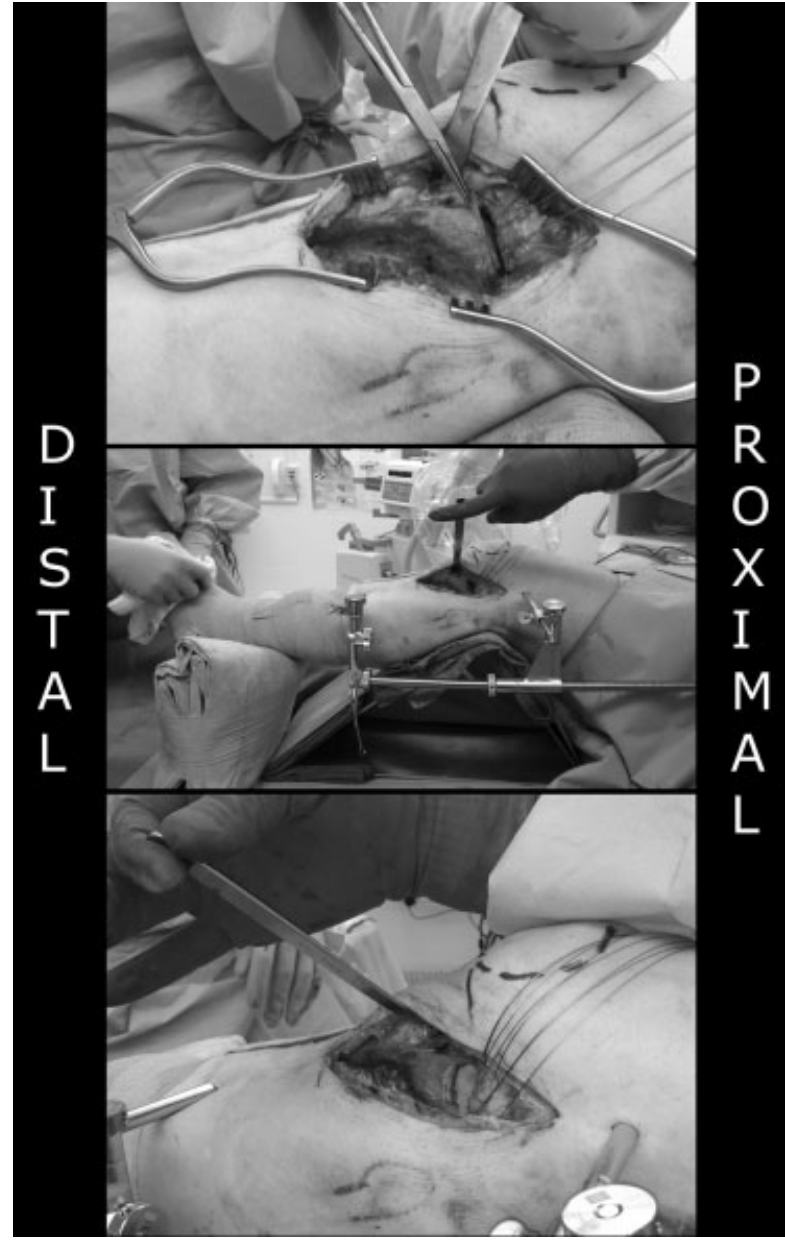

Fig. 3 Anterolateral approach: (Top) After submeniscal arthrotomy leaving a cuff of tissue on the tibial side, sutures are placed through the meniscus and capsule. (Middle) Application of the universal distractor. (Bottom) With application of distraction and retraction of the meniscocapsular tissue using sutures, the depressed joint surface is visualized.

pes tendons can be taken down for fracture reduction and later repaired. The broad insertion of the deep MCL (third layer ${ }^{10}$ ) makes arthrotomy and direct joint line visualization impractical. Indirect reduction is performed, and fixation is accomplished by direct application of plate over the MCL structures without elevation or mobilization.

\section{Additional Exposure}

This incision can be extended distally for a fasciotomy of the posterior compartments of the leg or for fixation of fracture extension into the tibial shaft as necessary.

\section{Posteromedial Approach}

This is an ideal approach for the typical shear fractures of medial tibia plateau, when the fracture line is in or close to the coronal plane and buttress plating is required with placement of fixation on the posterior or posteromedial surface of the medial tibial plateau. This approach is also used for shear fractures when the fracture line is in or close to the sagittal 


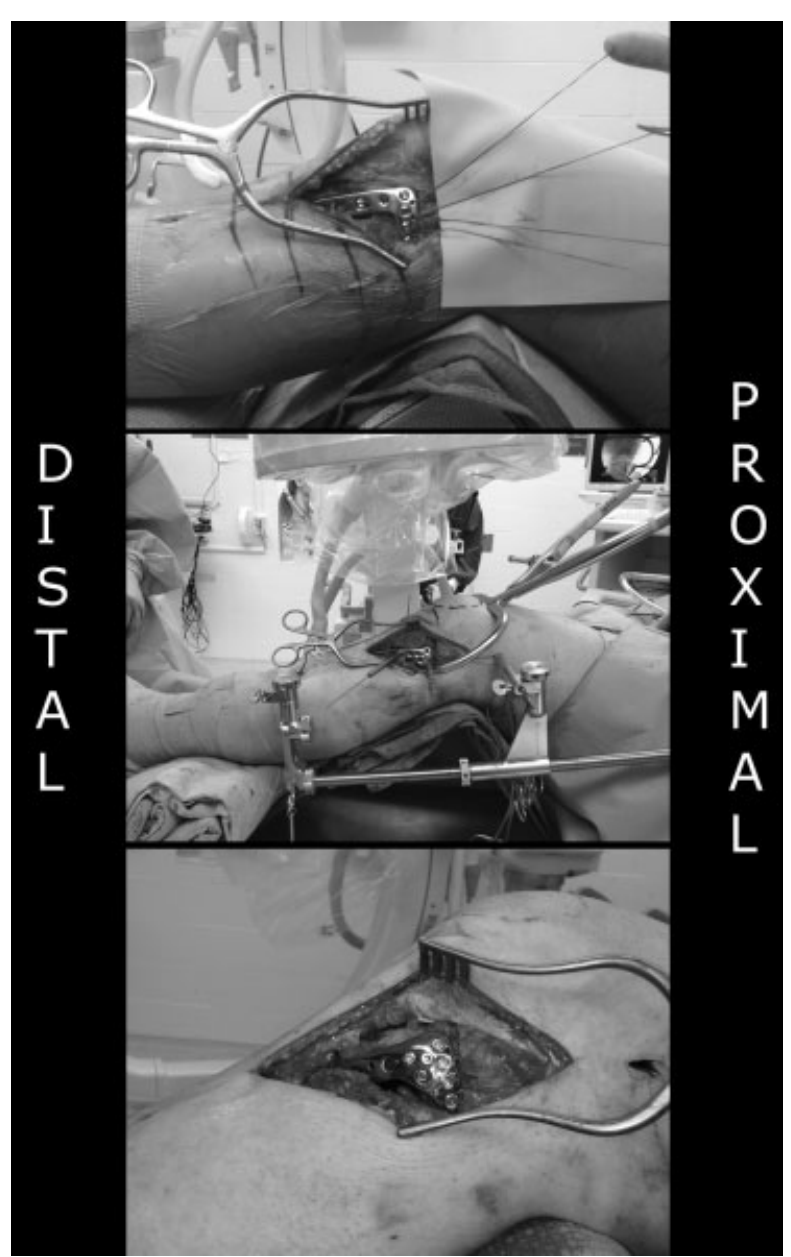

Fig. 4 Anterolateral approach: (Top) The sutures are passed through the Kwire holes in the periarticular plate. (Middle) Once the plate is apposed to the bone, a periarticular clamp is applied across the proximal tibia at the level of the subchondral bone through a small incision on the medial side and on one of the screw holes on the plate. (Bottom) Clamping assures the reduction of the width of the tibial plateau as well as compression across the fracture lines at the level of the joint.

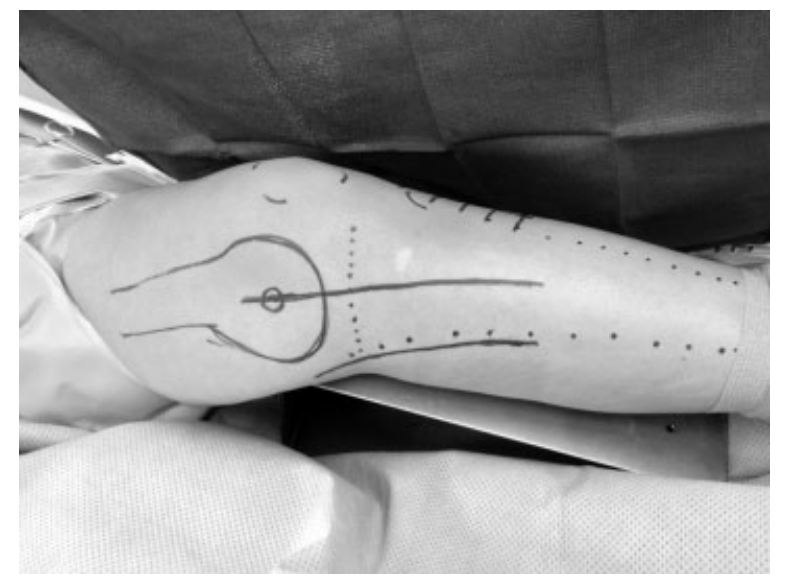

Fig. 5 Medial and posteromedial approaches. The skin incision for the medial approach starts 2 to $3 \mathrm{~cm}$ above joint line and is in line from the medial femoral epicondyle aiming bisecting posteromedial border of tibia and the tibial crest. The skin incision for the posteromedial approach starts 2 to $3 \mathrm{~cm}$ above joint line and follows the posteromedial border of tibia. plane and buttress plating is required with placement of fixation on the medial surface (at the junction of the posterior and anteromedial surface in cross section) of the tibial plateau. The Moore type I fracture has no specific designation in the Schatzker system, however, Barei et al found that nearly $40 \%$ of $41 \mathrm{C}$-type fractures have such a pattern. ${ }^{9}$ This approach can be done in the supine $\mathrm{e}^{11-13}$ or prone ${ }^{8,14}$ positioning. Prone positioning has the advantage of being ergonomic for the surgeon and easily permits the axial traction and extension/ hyperextension of the knee with the gravity that aids in indirect reduction. ${ }^{3,8}$ It is not recommended when there is involvement of the lateral plateau (in a dual approach strategy) as it requires repositioning of the patient, and adjustment to the initial fixation is not possible once the patient is turned supine for exposure of the fracture on the lateral plateau.

\section{Posteromedial Approach (Supine)}

\section{Superficial Dissection}

In the supine position with the leg externally rotated, a longitudinal incision along the posterior margin of the tibia is made (this is $1-2 \mathrm{~cm}$ more posterior than the medial approach) (-Fig. 6). ${ }^{12,13,15,16}$ The incision starts $3 \mathrm{~cm}$ proximal to the joint line and extends as far distally as needed. The saphenous nerve runs just anterior to the great saphenous vein. Both should be protected during exposure. Branches of the greater saphenous vein are ligated to retract it with the anterior or posterior flap. The sartorius fascia is incised in line with incision.

\section{Deep Dissection}

The fracture line exits commonly at the level of the pes tendons at the metadiaphysis. The pes anserinus tendons are mobilized and retracted distal-posterior or proximalanterior, whereas the medial gastrocnemius (MG) and soleus are retracted posteriorly. This exposes the junction between the popliteal fascia (posterior and distal), the semimembranosus (SM) insertion (posterior and proximal), and the MCL. Staying on the posterior border of the MCL, the periosteum is incised sharply longitudinally down to the bone. The proximal exposure is limited by the insertion of the SM. The SM is a broad attachment over the proximal posteromedial tibial plateau including continuity with the posterior medial meniscus. Using subperiosteal dissection, the popliteus muscle insertion is elevated off the posterior tibia to allow for direct visualization of the triangular apex of the fracture at the metadiaphyseal level, which allows indirect reduction of the joint and fixation with buttress plating. Subperiosteal dissection is critical as it avoids injury to the neurovascular structures including inferomedial genicular vessels. Reduction of the joint line is generally done indirectly by direct reduction of the apex and confirmed using fluoroscopic imaging, however, a posterior arthrotomy with elevation of the medial meniscus has been described. ${ }^{13,15,16}$ This arthrotomy requires extension of the subperiosteal dissection proximally, with elevation of the SM off the posteromedial tibia. Once the meniscotibial attachment is identified, a cuff is left on the tibia and stay sutures are placed for retraction and later 


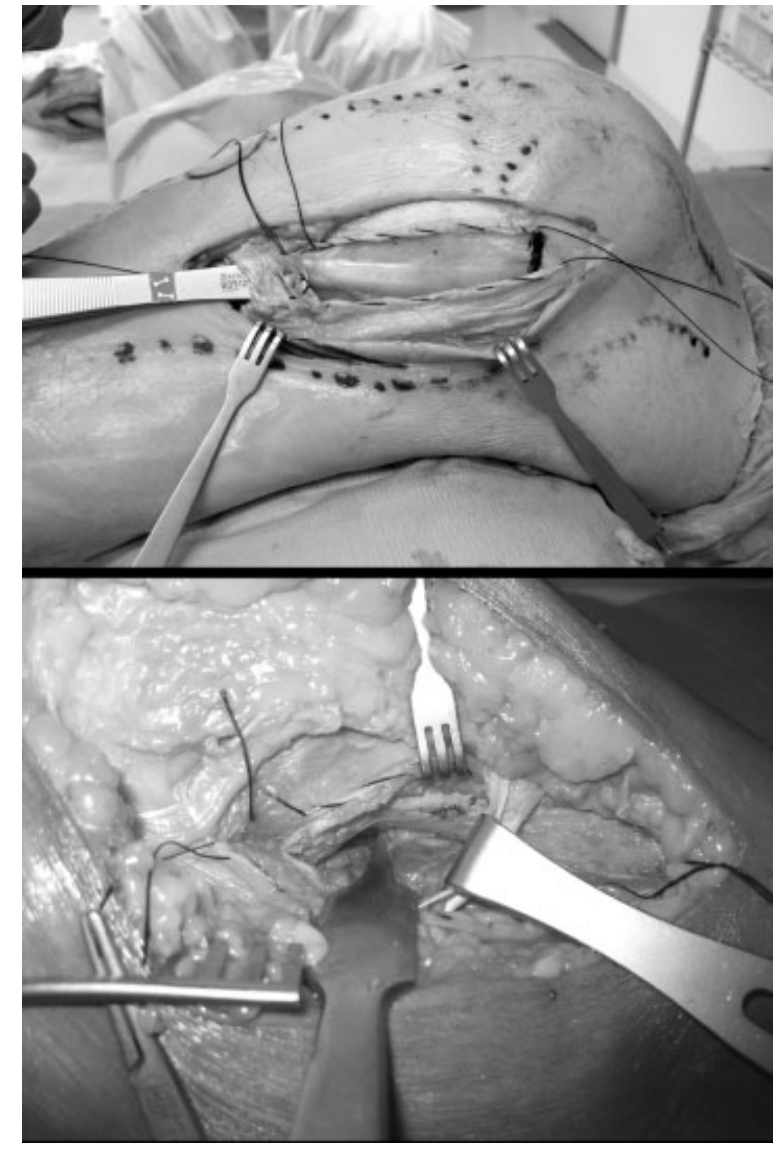

Fig. 6 Posteromedial approach: (Top) Incision of the sartorial fascia exposes the pes tendons, which are freed for retraction distal-posterior or proximal-anterior. (Bottom) The medial collateral ligament is incised at the posterior edge (retracted with a rake), the popliteus muscle insertion is reflected from the posterior border of tibia (retracted with Hohmann posteriorly), and the pes tendons is retracted distally exposing the common site of the fracture line exit at the metaphysis (marked).

repair. This is a far more limited visualization of the joint surface as compared with the lateral meniscal arthrotomy even with the use of a medially placed universal distractor. As most of the medial plateau fractures consist of simple fracture lines, indirect reduction of the joint line is the mainstay of our posteromedial approach. The reduction of the joint line is confirmed using fluoroscopic imaging. Assessment of the reduction can also be performed with arthroscopy. If direct visualization of the medial tibia plateau is deemed necessary for depression of joint surface, a medial parapatellar arthrotomy may provide a better exposure.

\section{Additional Exposure}

This incision can be extended distally for a fasciotomy of the posterior compartments of the leg or for fixation of fracture extension into the tibial shaft as necessary.

\section{Posteromedial Approach (Prone)}

This technique was first described in the German literature by Galla and Lobenhoffer, ${ }^{14}$ for treatment of isolated Moore type I tibial plateau fractures, as an alternative to the posterior approaches that traversed the popliteal fossa ( - Fig. 7). It was later described by Fakler et $\mathrm{al}^{8}$ in the English literature. The

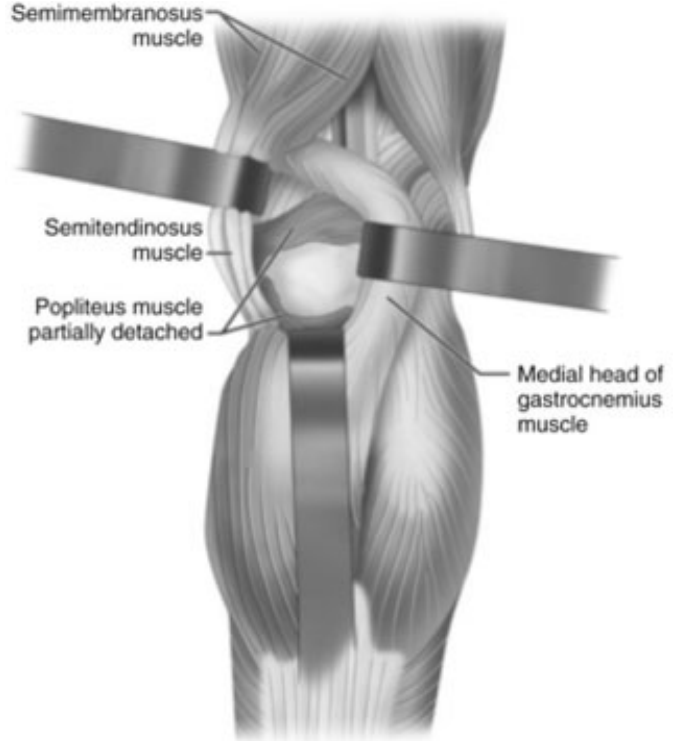

Fig. 7 Posteromedial approach in prone position: Retraction of the medial head of gastrocnemius laterally and pes tendons medially and reflection of the popliteus muscle insertion from the posterior medial proximal tibia laterally provides a direct view of the posteromedial tibia. (Reprinted with permission from Canale ST, Beaty JH. Campbell's Operative Orthopaedics. 12th ed. Copyright @ 2012 Mosby, An Imprint of Elsevier.)

patient is positioned prone with a small contralateral hip bump. A folded blanket is placed beneath the ipsilateral thigh to allow for hyperextension which, as noted by Moore, ${ }^{3}$ aids in the reduction of the fracture. A tourniquet is placed high on the thigh.

This approach is ideal for shear fractures of the posterior medial plateau when the fracture line is in the coronal or coronal-oblique plane. On the contrary, it should be limited to isolated fractures of the medial plateau because the adjustment of the reduction and fixation is not possible once the patient is turned supine for exposure of the lateral plateau.

\section{Superficial Dissection}

The skin incision is more posterior and lateral than that of the supine position. It is created longitudinally 8 to $10 \mathrm{~cm}$ in length starting just above the joint line and running along the medial border of the MG.

\section{Deep Dissection}

The MG is retracted laterally and is used to carefully protect the neurovascular structures that run deep and lateral to its muscle belly. The interval between the MG and the SM is developed without the need to dissect out the pes tendons anteriorly. The subperiosteal dissection is essentially the same, with sharp dissection of the popliteal fascia medially and subperiosteal elevation of the popliteus muscle off its insertion from the proximal posterior medial tibia starting from distal and proceeding proximally.

\section{Additional Exposure}

To gain additional lateral exposure, De Boeck and Opdecam described an "S"-type incision which starts proximally over 
the middle to lateral border of the MG, curves gently over the joint line and continues along the posteromedial border of the tibia. ${ }^{17}$ A partial release of the origin of the MG improves exposure of the posterior tibia. We reserve this approach for comminuted posterior/posteromedial fractures, and any instance in which the posterior cruciate ligament (PCL) is compromised (as reconstruction can be done from this extended exposure ${ }^{11}$ ).

\section{Posterolateral Approach}

The principal indication for this approach is a coronal fracture line resulting in a displaced posterolateral fragment (-Fig. 8). ${ }^{7,18,19}$ This approach should be reserved to fracture patterns that cannot be addressed through the anterolateral approach because of the risk of complications including stiffness and peroneal nerve injury. Posterolateral fractures of tibial plateau are not specifically accounted for in the common classification systems. Although described as "rare" injury patterns, retrospective studies have observed a 7 to $10 \%$ incidence in operatively managed proximal tibial fractures. ${ }^{19-21}$ Solomon et al reviewed nine such cases treated by way of an anterior or anterolateral approach, and noted that none achieved anatomic reduction. ${ }^{19}$ They subsequently changed techniques, and a retrospective analysis at 2-year follow-up demonstrated superior clinical and radiographic outcomes with the use of a posterolateral approach. ${ }^{22}$ Extensive soft tissue dissection is required, however, and flexion contractures of 5 to 10 degrees have been seen in upward of $50 \%$ of the patients. ${ }^{18,23}$

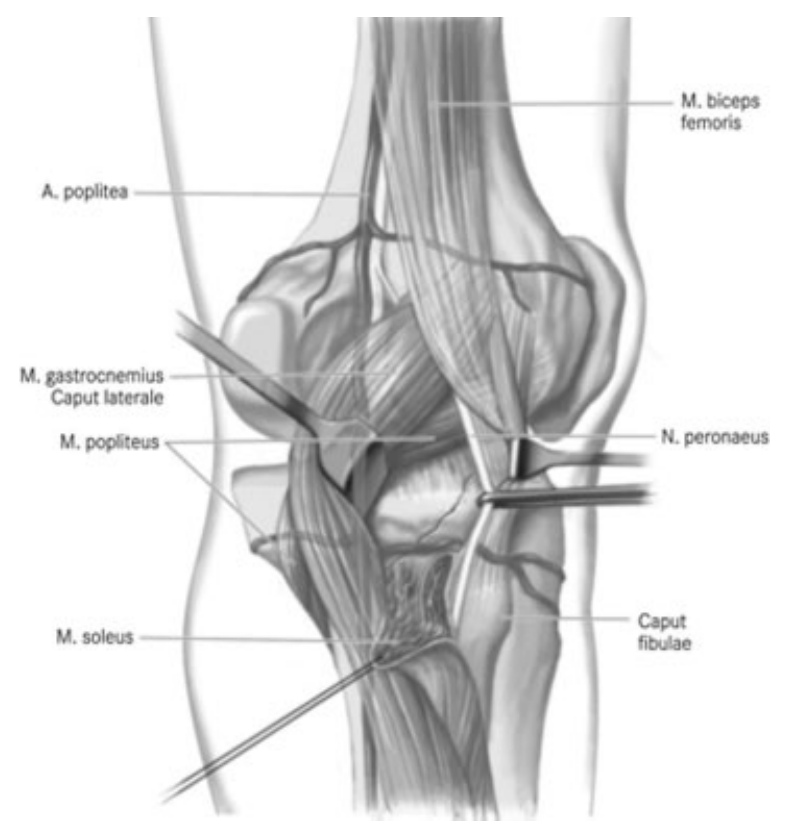

Fig. 8 Posterolateral approach without fibular osteotomy: This approach should be limited to fractures that cannot be addressed through other approaches such as shear fractures of the posterolateral plateau amenable for buttress plating. (Reprinted from Frosch KH, Balcarek P, Walde T, Stürmer KM. A new posterolateral approach without fibula osteotomy for the treatment of tibial plateau fractures. J Orthop Trauma 2010;24(8):515520, with permission from Lippincott Williams and Wilkins.)
The technique was described by Lobenhoffer et al in $1997^{7}$ and numerous variations have appeared since then, the principal difference being the presence ${ }^{7,19}$ or absence $^{15,18,23-25}$ of a fibular osteotomy. Proponents of the osteotomy cite the improved exposure of the lateral and posterolateral joint line as the principal advantage ${ }^{19}$; however, dissection of the peroneal muscle origin, posterolateral ligamentous structures, and need for osteotomy repair are all additional sources of complication. ${ }^{26}$ As such, we use the fibular sparing approach as described by Carlson. ${ }^{24}$ An excellent English description of the osteotomy technique is published ${ }^{19}$ and will not be described here.

The patient can be positioned in the prone, $7,18,23,24$ supine, ${ }^{19}$ or lateral position. ${ }^{25}$ Supine positioning is more technically demanding and requires the use of a sand bag or foot holder to flex the knee without blocking the popliteal fossa. We reserve this for the rare instance when additional anterior intervention is required, as in the case of an ipsilateral shaft fracture requiring intramedullary nailing.

\section{Positioning}

A thigh tourniquet is applied before draping. The patient is placed in the prone position on bolsters. A small bump is placed under the operative thigh. This elevates the limb for lateral radiographs and allows for hyperextension during reduction.

\section{Superficial Dissection}

A straight $10-\mathrm{cm}$ longitudinal incision is made from the medial aspect of the biceps femoris (BF) tendon proximally to the posteromedial border of the fibula distally. Dissection is carried through skin, subcutaneous tissue, and popliteal fascia. The interval between the BF and lateral gastrocnemius (LG) muscle is palpated. At the proximal most aspect of this, the fat streak containing the common peroneal nerve (CPN) can be identified just medial to the BF. The CPN gives off the lateral sural cutaneous nerve ( $\mathrm{LSCN}$ ) at this level. The plane of dissection is between the $\mathrm{LG}$, which will be retracted medially with the LSCN, and the BF which will be retracted laterally with the CPN. The CPN runs along the inferior medial border of the BF as it travels distally. It perforates the posterior intermuscular septum just below the insertion of the BF on the fibula and dives into the peroneal tunnel. The nerve must be mobilized over the entire length and retracted laterally for protection.

\section{Deep Dissection}

The LG is the limiting factor for medial exposure. Depending on the fracture pattern, its origin can be left intact, partially elevated (from inferior lateral to superior medial), or released and repaired later. Distally, the soleus is encountered at its origin on the posterolateral tibia and fibula. Blunt elevation of the soleus will provide exposure of the proximal tibia. Distal exposure should be limited to 4 to $5 \mathrm{~cm}$ below the joint line to avoid injury to the anterior tibial artery, which travels laterally through the intermuscular septum to the anterior compartment (approximately 5-7 cm below the joint line). Care is also taken when releasing the soleus from the fibular 
neck to avoid injury to the CPN. The popliteus muscle and tendon are usually coursing over the proximal joint line at the level of the fracture. Before mobilizing this structure, the inferolateral genicular artery should be identified. It runs just inferior to the popliteus muscle-tendon structure and travels deep to the fibular collateral and popliteus-fibular ligaments as it courses anteriorly. If observed, the vessel should be ligated before proceeding. Finally, the posterior capsuloligamentous complex is incised from medial to lateral. Stay sutures are placed for retraction and later repair.

\section{Additional Exposure}

Posterolateral fractures can occur in combination with the common central and/or anterior fractures of the lateral plateau. In these instances, the subcutaneous dissections of the anterolateral and posterolateral approaches can be combined through a single incision as described by Frosch et al. ${ }^{25}$ The skin incision is made directly along the femoral condyle and the proximal fibula (bisecting between that of the anterolateral and posterolateral exposures). The deep dissection is performed as described above. In this manner, the CPN will lie between the two surgical exposures, and extra care must be taken to avoid iatrogenic injury.

\section{Direct Posterior Approach}

Because of the risk of iatrogenic injury to neurovascular structures in the popliteal fossa and risk of flexion contracture, the direct posterior approach should be limited to the fracture patterns that cannot be addressed with posteromedial and/or posterolateral approaches (-Fig. 9). The injury pattern most commonly treated with this approach is a shear fracture of the posterior plateau with main fracture line in coronal plane or avulsion of PCL insertion with a fracture line extending into the large articular surface. The original ap- proach was described by Abbott and Carpenter in $1945^{27}$ as a midline approach with careful dissection of all popliteal structures. Over time, several variations including a MG splitting, ${ }^{28}$ "S-"shaped curvilinear, ${ }^{29}$ and medially based inverted "L-"type incision ${ }^{11}$ have been described for treatment of soft tissue abnormalities (PCL injury, Baker cyst, or pigmented villonodular synovitis); however, only one report focuses specifically on the use of this approach for management of posterior tibial plateau fractures. ${ }^{20}$ A detailed, pictographic, description of the landmarks and relative anatomy of the posterior approach in a cadaver series was also recently published. ${ }^{30}$

\section{Superficial Dissection}

An "S-"shaped incision is created along the BF tendon proximally, then curving across the popliteal fossa at the joint line and distally along the medial head of the gastrocnemius muscle. The incision is made carefully through the skin only. At, or just below, the joint line the lesser saphenous vein and sural nerve are identified piercing through the popliteal fascia. The sural nerve can be followed proximally and used as a landmark for safely identifying the tibial nerve. Then, the fascia between the sural nerve and medial head of the gastrocnemius is incised. The tibial nerve is followed proximally to the point where the SM and BF meet. On the medial edge of the BF lies the CPN which should be mobilized and retracted laterally.

\section{Deep Dissection}

The vascular structures run medial to the tibial nerve. The popliteal vein is lateral to the artery at the level of the femur, crosses superficial to it at the joint line, and runs medial to it along the tibia. Depending on the fracture pattern and exposure required, one or more of the five genicular branches may need to be ligated for adequate exposure and mobilization of

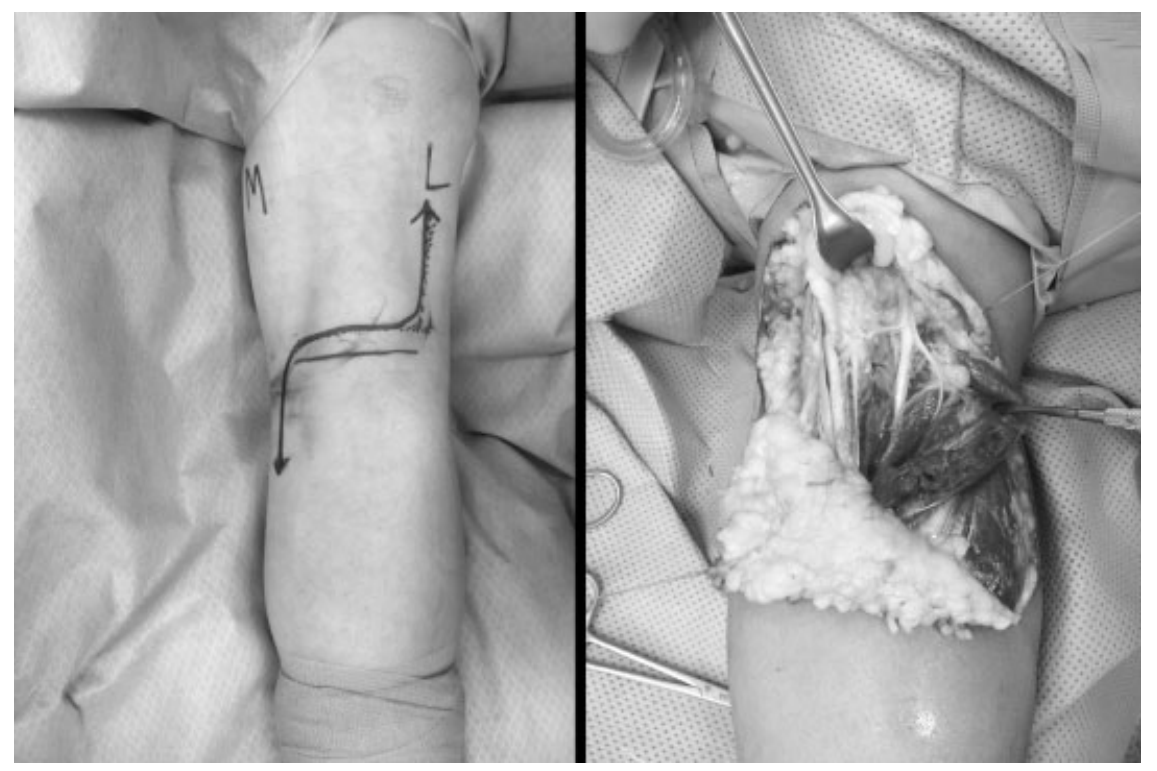

Fig. 9 Direct posterior approach: (Left) An "S-"shaped incision is created along the biceps femoris tendon proximally, then curving across the popliteal fossa at the joint line and distally along the medial head of the gastrocnemius muscle. (Right) Lesser saphenous vein and medial cutaneous sural nerve is on the medial side, tibial nerve is in the middle with retraction of the lateral head of the gastrocnemius. 
the popliteal artery. If either head of the gastrocnemius is mobilized, the corresponding superior genicular artery may need to be ligated. Flexion of the knee can reduce tension on the medial genicular artery and avoid injury to this vessel when working near the center of the joint line.

The deep dissection is the same as that described above for the posteromedial and posterolateral approaches, with mobilization of the MG, or detachment of the LG and mobilization of the BF.

\section{Additional Exposure}

When concern for vascular injury necessitates, the distal aspect of the incision can be extended and the terminal divisions of the popliteal artery can be identified superficial to the popliteus muscle.

\section{Summary}

An ideal surgical approach should allow visualization and reduction of the fracture and application of fixation with minimal risk of complications such as neurovascular injury and wound-healing problems. Although the anterolateral approach is the most commonly applied technique, having the knowledge of different approaches in the armamentarium is necessary to treat the vast array of fracture patterns that occur in the proximal tibia. Dual approaches combining anterolateral and posteromedial exposures are the mainstay choice for most bicondylar tibial plateau fractures. A detailed analysis of the fracture pattern should dictate the choice of the appropriate approach for specific fractures.

\section{References}

1 Chan PS, Klimkiewicz JJ, Luchetti WT, et al. Impact of CT scan on treatment plan and fracture classification of tibial plateau fractures. J Orthop Trauma 1997;11(7):484-489

2 Fernandez DL. Anterior approach to the knee with osteotomy of the tibial tubercle for bicondylar tibial fractures. J Bone Joint Surg Am 1988;70(2):208-219

3 Moore TM. Fracture-dislocation of the knee. Clin Orthop Relat Res 1981;156(156):128-140

4 Moore TM, Patzakis MJ, Harvey JP. Tibial plateau fractures: definition, demographics, treatment rationale, and long-term results of closed traction management or operative reduction. J Orthop Trauma 1987;1(2):97-119

5 Tscherne H, Lobenhoffer P. Tibial plateau fractures. Management and expected results. Clin Orthop Relat Res 1993;292(292): 87-100

6 Padanilam TG, Ebraheim NA, Frogameni A. Meniscal detachment to approach lateral tibial plateau fractures. Clin Orthop Relat Res 1995;314(314):192-198

7 Lobenhoffer P, Gerich T, Bertram C, Lattermann T, Pohlemann H, Tscherne H. Treatment of posterior tibial plateau fractures via posteromedial and posterolateral exposures. Unfallchirurg 1997; 100(12):957-967

8 Fakler JK, Ryzewicz M, Hartshorn C, Morgan SJ, Stahel PF, Smith WR. Optimizing the management of Moore type I postero-medial split fracture dislocations of the tibial head: description of the Lobenhoffer approach. J Orthop Trauma 2007;21(5):330-336
9 Barei DP, O’Mara TJ, Taitsman LA, Dunbar RP, Nork SE. Frequency and fracture morphology of the posteromedial fragment in bicondylar tibial plateau fracture patterns. J Orthop Trauma 2008; 22(3):176-182

10 Warren LA, Marshall JL, Girgis F. The prime static stabilizer of the medical side of the knee. J Bone Joint Surg Am 1974;56(4): 665-674

11 Burks RT, Schaffer JJ. A simplified approach to the tibial attachment of the posterior cruciate ligament. Clin Orthop Relat Res 1990; 254(254):216-219

12 Georgiadis GM. Combined anterior and posterior approaches for complex tibial plateau fractures. J Bone Joint Surg Br 1994;76(2): 285-289

13 Weil YA, Gardner MJ, Boraiah S, Helfet DL, Lorich DG. Posteromedial supine approach for reduction and fixation of medial and bicondylar tibial plateau fractures. J Orthop Trauma 2008;22(5): 357-362

14 Galla M, Lobenhoffer P. [The direct, dorsal approach to the treatment of unstable tibial posteromedial fracture-dislocations]. Unfallchirurg 2003;106(3):241-247

15 Carlson DA. Posterior bicondylar tibial plateau fractures. J Orthop Trauma 2005;19(2):73-78

16 Wang SQ Gao YS, Wang JQ Zhang CQ Mei J, Rao ZT. Surgical approach for high-energy posterior tibial plateau fractures. Indian J Orthop 2011;45(2):125-131

17 De Boeck H, Opdecam P. Posteromedial tibial plateau fractures. Operative treatment by posterior approach. Clin Orthop Relat Res 1995;320(320):125-128

18 Tao J, Hang DH, Wang QG, et al. The posterolateral shearing tibial plateau fracture: treatment and results via a modified posterolateral approach. Knee 2008;15(6):473-479

19 Solomon LB, Stevenson AW, Baird RP, Pohl AP. Posterolateral transfibular approach to tibial plateau fractures: technique, results, and rationale. J Orthop Trauma 2010;24(8):505-514

20 Bhattacharyya T, McCarty LP III, Harris MB, et al. The posterior shearing tibial plateau fracture: treatment and results via a posterior approach. J Orthop Trauma 2005;19(5):305-310

21 Partenheimer A, Gösling T, Müller M, et al. [Management of bicondylar fractures of the tibial plateau with unilateral fixedangle plate fixation]. Unfallchirurg 2007;110(8):675-683

22 Solomon LB, Stevenson AW, Lee YC, Baird RP, Howie DW. Posterolateral and anterolateral approaches to unicondylar posterolateral tibial plateau fractures: a comparative study. Injury 2013;44(11): 1561-1568

23 Chang SM, Zheng HP, Li HF, et al. Treatment of isolated posterior coronal fracture of the lateral tibial plateau through posterolateral approach for direct exposure and buttress plate fixation. Arch Orthop Trauma Surg 2009;129(7):955-962

24 Carlson DA. Bicondylar fracture of the posterior aspect of the tibial plateau. A case report and a modified operative approach. J Bone Joint Surg Am 1998;80(7):1049-1052

25 Frosch KH, Balcarek P, Walde T, Stürmer KM. A new posterolateral approach without fibula osteotomy for the treatment of tibial plateau fractures. J Orthop Trauma 2010;24(8):515-520

26 Hsieh CH. Treatment of the Posterolateral Tibial Plateau Fractures using the Anterior Surgical Approach. Int J Biomed Sci 2010;6(4): 316-320

27 Abbott LC, Carpenter WF. Surgical approaches to the knee joint. J Bone Joint Surg Am 1945;27(2):277-310

28 Trickey EL. Rupture of the posterior cruciate ligament of the knee. J Bone Joint Surg Br 1968;50(2):334-341

29 Hughston JC. A surgical approach to the medial and posterior ligaments of the knee. Clin Orthop Relat Res 1973;91(91):29-33

30 Alpert JM, McCarty LP, Bach BR Jr. The direct posterior approach to the knee: surgical and anatomic approach. J Knee Surg 2008;21(1): $44-49$ 